Article

\title{
Placenta-Derived Mesenchymal Stem Cells Restore the Ovary Function in an Ovariectomized Rat Model via an Antioxidant Effect
}

\author{
Jin Seok ${ }^{1 \oplus}$, Hyeri Park ${ }^{1} \oplus$, Jong Ho Choi ${ }^{2}{ }^{\oplus}$, Ja-Yun Lim ${ }^{3} \oplus$, Kyung Gon Kim ${ }^{4}{ }^{\oplus}$ and \\ Gi Jin Kim $1, * \mathbb{D}$ \\ 1 Department of Biomedical Science, CHA University, Seongnam 13488, Korea; jjin8977@gmail.com (J.S.); \\ hyeyeyeri@gmail.com (H.P.) \\ 2 Department of Oral Pathology, Gangneung-Wonju National University, Gangneung 26403, Korea; \\ jhchoi@gwnu.ac.kr \\ 3 Department of Integrated Biomedical and Life Sciences, College of Health Science, Korea University, \\ Seoul 02841, Korea; jayun78@korea.ac.kr \\ 4 Department of Convergence Medicine, School of Medicine, University of Ulsan and Asan Medical Center, \\ Seoul 05505, Korea; kimkyunggon@gmail.com \\ * Correspondence: gjkim@cha.ac.kr; Tel.: +82-31-881-7145
}

Received: 12 June 2020; Accepted: 3 July 2020; Published: 6 July 2020

\begin{abstract}
Oxidative stress is one of the major etiologies of ovarian dysfunction, including premature ovarian failure (POF). Previous reports have demonstrated the therapeutic effects of human placenta-derived mesenchymal stem cells (PD-MSCs) in an ovariectomized rat model (OVX). However, their therapeutic mechanism in oxidative stress has not been reported. Therefore, we investigated to profile the exosome of serum and demonstrate the therapeutic effect of PD-MSCs transplantation for the ovary function. We established an OVX model by ovariectomy and PD-MSCs transplantation was conducted by intravenous injection. Additionally, various factors in the exosome were profiled by LC-MS analysis. As a result, the transplanted PD-MSCs were engrafted into the ovary and the existence of antioxidant factors in the exosome. A decreased expression of oxidative stress markers and increased expression of antioxidant markers were shown in the transplantation (Tx) in comparison to the non-transplantation group (NTx) $\left({ }^{*} p<0.05\right)$. The apoptosis factors were decreased, and ovary function was improved in Tx in comparison to NTx $\left({ }^{*} p<0.05\right)$. These results suggest that transplanted PD-MSCs restore the ovarian function in an OVX model via upregulated antioxidant factors. These findings offer new insights for further understanding of stem cell therapy for reproductive systems.
\end{abstract}

Keywords: premature ovarian failure; ovariectomized rat model; stem cell therapy; placenta-derived mesenchymal stem cells; folliculogenesis; reactive oxidative stress; antioxidants

\section{Introduction}

The ovary is a specialized organ that ensures reproductive success during a definite life stage as the main regulator of female fertility. However, the ovaries are more severely affected by age compared to other organs. Ovarian aging results in ovarian failure and menopause and leads to infertility through the reduction in important sex hormones, follicle loss, and oocyte quality decline [1]. In recent studies, ovarian dysfunction has been shown to increase due to genetic factors (e.g., genomic DNA alteration, mitochondrial DNA mutations, and decreased telomerase activity), as well as various environmental factors (e.g., oxidative stress, advanced glycation, and products) [2]. These processes of ovarian dysfunction accelerate with aging and increase as women age. In particular, POF is the loss of 
function of the ovaries in women under the age of 40. It is characterized by a decrease in the function of the ovary, which is similar to the menopausal period, but it is different from a general menopausal period. It is defined as a low E2 concentration and high FSH hormone, and it is characterized by various degenerative diseases, including heart disease [3-5].

Recently, hormone replacement therapy (HRT) and cryopreservation have been applied to patients as an alternative to overcome ovarian dysfunction. HRT is widely employed as a hormone control method, but it does involve a risk of illness, such as heart disease, stroke, breast cancer, and colorectal cancer, in patients. However, it has recently been reported that for women under the age of 60 , the various risks can be reduced when it is used at an appropriate time. However, hormone therapy has the disadvantage of being ineffective when HRT is stopped or not used continuously [6,7]. Ovarian tissue cryopreservation is a new therapy employed to treat women with POF, but it has a lower survival rate of the thawed ovary, including difficulties with conceiving naturally [8]. Therefore, it is important to develop safe new treatments that can recover ovary dysfunction.

PD-MSCs are reported to have multiple abilities, including strong self-renewal, multipotent differentiation, and immunomodulatory properties $[9,10]$. In addition, PD-MSCs secrete more various cytokines, such as growth factors, compared to other stem cells (e.g., bone marrow-derived MSCs (BM-MSCs) and adipose-derived MSCs (AD-MSCs)). Lee et al. and their colleagues demonstrated that PD-MSCs secrete various cytokines, including G-CSF, RANTES, and IL-6/-8/-10 [11]. Interestingly, several studies have shown that these cytokines also affect the mitochondrial function in cells. Savitree et al. suggested that granulocyte-colony stimulating factors (G-CSF), which are mitochondria-dependent caspase 3 inhibitors, directly protect cardiac mitochondria from oxidative stress [12]. The potential of PD-MSCs has been highlighted in various degenerative diseases due to their therapeutic effects as a new stem cell therapy [13-18]. In previous reports, we reported that a 3D spheroid from human PD-MSCs stimulates folliculogenesis, as well as engraftment into the ovary tissues, in an ovarian failure rat model with ovariectomy (OVX) [19].

Exosomes are small extracellular vesicles with a diameter size range of -40 to $160 \mathrm{~nm}$ that are secreted from most human cell types and contain a specific composition of protein, lipid, RNA, and DNA [20]. Isolated exosomes, known as markers (such as the tetraspanins family (CD63, CD9, CD81, and CD82) and heat shock proteins (HSPs; HSP60, HSP70, and HSP90) have been characterized [21]. It is proved that exosomes can have various effects in vivo depending on the microenvironment and location of production. Especially, exosomes play a role in cell to cell communication and have been in the spotlight for their clinical applications for various diseases via changed microenvironments [22]. Several researchers have demonstrated that exosomes changed the microenvironment and indicated biomarkers in ovarian cancer, as well as other severe diseases [23,24]. It is reported that secreted exosome by oxidative stress prevents cell death for defense mechanisms [25]. Hence, many studies have been reported on mechanisms using exosomes from stem cells in diseases. However, the study is still insufficient to determine whether the stem cells affect the microenvironment, including exosomes.

Reactive oxygen species (ROS), such as molecules of superoxide, hydroxyl radicals, hydrogen peroxide, and hypohalous acids, are generated by $\mathrm{H}_{2} \mathrm{O}_{2}$ and the NADPH oxidase system during cellular metabolism and removed by antioxidants such as superoxide dismutase, glutathione peroxidase (GPX), and catalase (CAT) [26,27]. Moreover, it is known that oxidative stress is increased in damaged cells/tissues and induces damage. At this time, the mechanism for deciphering the reactive intermediates is activated to recover and regenerate the damaged cells and maintain homeostasis of the body [28,29]. A previous study demonstrated that ROS activate p53 and upregulate the expression level of the pro-apoptotic proteins of BAX and PUMA, resulting in cell apoptosis [30]. In the ovary, ROS are generated during follicular rupture, which is an inflammation-like reaction, and oocyte ovulation [31]. However, excessively accumulated ROS trigger infertility by a loss of oocyte maturation and granulosa cell luteinization $[32,33]$. Several recent studies have reported that increases in the ROS level reduce the oocyte quality, reproductive outcome, and granulosa cell apoptosis [34,35]. However, because there is still a lack of research, understanding and studying the correlation between oxidative stress 
and ovarian dysfunctional disorder is necessary. Therefore, in this study, we aimed to investigate the therapeutic effect of PD-MSCs via antioxidant signaling in an OVX rat model.

\section{Materials and Methods}

\subsection{Animals}

All experiments involving animals were performed in accordance with the animal care guidelines issued by the National Institutes of Health and were approved by the Institute Animal Care and Committee of the CHA Laboratory Animal Research Center at Sampyeng-dong in Gyeonggi, Korea (IACUC 190048). All female Sprague-Dawley rats were purchased from Orient Bio Inc. (Orient Bio Inc., Seongnam, Gyeonggi, Korea) and were 8 weeks old in this study. The rats were housed in groups of two rats per plastic cage with corn-cob bedding and were provided with ad libitum access to standard commercial food and tap water. The temperature was $21^{\circ} \mathrm{C}$, and a $12 \mathrm{~h} / 12 \mathrm{~h}$ light-dark cycle was employed.

\subsection{Ovariectomized Rat Model Establishment}

All animal experiments were approved by the Institutional Animal Care and Use Committee (IACUC 190048) of the CHA Laboratory Animal Research Center (Gyeonggi-do, Korea). The following week, 40 animals were randomly allocated into two groups. The NTx group included ovariectomized $(\mathrm{OVX})$ rats $(n=20)$, and the Tx group included indirectly stem cell transplanted OVX rats obtained through a tail vein injection $(n=20)$. Ovariectomy was performed in female rats of all groups to remove one of the ovaries. All rats were anesthetized via intraperitoneal injection with $250 \mathrm{mg} / \mathrm{kg}$ avertin (Sigma-Aldrich, St. Louis, MO, USA). After all rats had been sterilized using $70 \%$ ethanol with distilled water, the skin and muscles in the pelvic area of the back were incised and the tissue of one ovary was tied off with a sterile suture and removed. After removal of the ovary, the surgical site was disinfected with povidone-iodine (Sigma-Aldrich, St. Louis, MO, USA) and all OVX rats were maintained in their housing cages for one week.

\subsection{Cell Culture of PD-MSCs and Transplantation into an Ovariectomized Rat Model}

Placentas were collected from women who were free of any medical, obstetrical, or surgical complications and who delivered at term ( $38 \pm 2$ gestational weeks). PD-MSCs were isolated from human placental chorionic plates and approved by the Institutional Review Board of CHA General Hospital, Seoul, Korea (IRB 07-18). PD-MSCs were isolated from chorionic plates of normal-term placentas, as previously described by Lee et al. [13]. Briefly, PD-MSCs were cultured in alpha-minimum essential medium ( $\alpha$-MEM; Hyclone, GE healthcare life sciences, Seoul, Korea) supplemented with $10 \%$ fetal bovine serum (FBS; Gibco-BRL, Rockville, MD, USA), 1\% penicillin/streptomycin (Pen-Strep; Gibco-BRL), 25 mg/mL human fibroblast growth factor 4 (hFGF-4; Peprotech Inc., Rocky Hill, NJ, USA), and $1 \mu \mathrm{g} / \mathrm{mL}$ heparin (Sigma-Aldrich) at $37^{\circ} \mathrm{C}$ in an incubator with a humidified atmosphere of $5 \%$ $\mathrm{CO}_{2}$. One week after the ovariectomy, PD-MSCs $\left(5 \times 10^{5}\right)$ were labeled using a PKH67 Fluorescent Cell linker kit (Sigma-Aldrich) and injected through the tail vein. After blood samples had been collected for hormone level analysis, the rats of all groups were sacrificed, and ovary tissues were harvested at 1 , 2,3 , and 5 weeks using liquid nitrogen. All ovary tissues and blood samples of each group (NTx and $\mathrm{Tx} ; 1,2,3$, and 5 weeks; $n=5$ ) were pooled to ensure there was no variation between the groups.

\subsection{Exosome Sample Preparation for Proteome Analysis}

To analyze the exosome of the serum in the OVX rat model, we isolated the exosome using a precipitation kit (System Biosciences, Palo Alto, CA, USA), following the manufacturer's instructions. Protein amounts of the isolated exosome samples were measured using a bicinchoninic acid (BCA) assay and $100 \mu \mathrm{g}$ of each protein was taken and dried. Each sample was lysed in $300 \mu \mathrm{L}$ of lysis buffer consisting of $5 \%$ sodium dodecyl sulfate and $50 \mathrm{mM}$ triethylammonium bicarbonate ( $\mathrm{pH} 7.55$, Thermo 
Fisher Scientific, Waltham, MA, USA) by sonication on ice. The lysates were cleared by centrifugation at 15,000 rpm for $15 \mathrm{~min}$ at $4{ }^{\circ} \mathrm{C}$. Each sample underwent STrap-based tryptic digestion employing previously known methods [36] using a trypsin/LysC mixture (Promega, Madison, WV, USA).

\subsection{Nano-LC-ESI-MS/MS Analysis}

Samples were analyzed on a Dionex UltiMate 3000 RSLC nano LC system (Thermo Scientific, Waltham, MA, USA) coupled to a Q Exactive plus mass spectrometer (Thermo Scientific) with a nano-ESI source. Tryptic peptides from a bead column were reconstituted using $0.1 \%$ formic acid and were loaded via an Acclaim PepMap 100 trap column $(100 \mu \mathrm{m} \times 2 \mathrm{~cm}$, nanoViper, C18, $5 \mu \mathrm{m}$, $100 \AA$, Thermo Scientific). Subsequent peptide separation was performed on an Acclaim PepMap rapid separation LC (RSLC) analytical column $(75 \mu \mathrm{m} \times 50 \mathrm{~cm}$, nanoViper, C18, $2 \mu \mathrm{m}, 100 \AA$, Thermo Scientific) for over $200 \mathrm{~min}(250 \mathrm{~nL} / \mathrm{min})$ using a $0 \%$ to $40 \%$ acetonitrile gradient in $0.1 \%$ formic acid at $50{ }^{\circ} \mathrm{C}$. Mass spectra were acquired in a data-dependent mode with automatic switching between a full scan $(m / z$ 350-1800) and 20 data-dependent MS/MS scans. The target value for the full-scan MS spectra was 3,000,000, with a maximum injection time of $100 \mathrm{~ms}$ and a resolution of 70,000 at $\mathrm{m} / \mathrm{z} 400$. The ion target value for MS/MS was set to 100,000 with a maximum injection time of $50 \mathrm{~ms}$ and a resolution of 17,500 at $m / z 400$ with normalized collision energy (27\%). The dynamic exclusion of repeated peptides was applied for $20 \mathrm{~s}$. For each biological sample, three technical replicates were performed.

\subsection{Database Search and Label-Free Quantitation and staTistical Analysis}

The acquired MS/MS spectra were searched using the SequestHT on Proteome discoverer (version 2.2, Thermo Fisher Scientific) against the SwissProt database (June 2019). Briefly, the precursor mass tolerance was set to $\pm 10 \mathrm{ppm}$ and the MS/MS tolerance was set to $0.02 \mathrm{Da}$. The search parameters were set as default, including cysteine carbamidomethylation (CAM) as a fixed modification, and $\mathrm{N}$-terminal/lysine acetylation, $\mathrm{N}$-terminal methionine loss, and acetylation, methionine oxidation, phosphoserine, phosphor-threonine, and phosphor-tyrosine as variable modifications with two miscleavages. False discovery rates (FDRs) were set to $1 \%$ for each analysis using "Percolator". From the SequestHT search output, the peptide filters for peptide confidence, peptide rank, score versus charge state, and search engine rank were applied at the default values of the Proteome discoverer. Label-free quantitation was performed using the peak intensity for unique and razor peptides of each protein. Normalization was conducted using the total peptide amount. For differential analysis of the relative abundance of proteins between samples, the free software Perseus (version 1.6.10.43; Maxquant, Germany) was used. The values of normalized protein abundances were transformed into log2 scale values. Technical replicates of each sample were grouped and a minimum of three valid values was required in at least one group. To find statistically significant differences between samples, a t-test was performed using permutation-based FDR ( 0.05 cut-off). The hierarchical clustering of enrichment z-score values was performed using InstantClue software (http://www.instantclue.uni-koeln.de) [37]. Hierarchical clustering was performed using the Euclidean distance as a metric and the average linkage. The quantitation data were uploaded into the Ingenuity Pathways Analysis (IPA; Ingenuity Systems, Redwood City, CA, USA) program. Data were analyzed through the Ingenuity Pathway Analysis platform (QIAGEN Inc., Valencia, CA, USA). Proteins in the proteomic data were mapped to corresponding gene objects in the Ingenuity Pathways knowledge base. Then, biological networks were generated using the knowledge base for interactions between the uploaded gene list and all other gene objects stored in the knowledge base. A functional analysis of the networks was conducted to identify the biological functions and/or diseases that were most significant to the genes in the network. The score was derived from the $\mathrm{p}$-value of the test and indicated the likelihood of the mapped genes in a network being found together due to random chance (score $=-\log _{10} p$ ). 


\subsection{Hormone ELISA Assay}

All blood samples were collected from the aorta in rats of NTx and Tx groups via the recto-orbital technique. The serum samples were separated from whole blood by using a blood collection tube (vacutainer; BD Biosciences, San Jose, CA, USA) at 1300 RCF for $15 \mathrm{~min}$. All blood serum was stored at $-80^{\circ} \mathrm{C}$ and the estrogen (Bio vision, Milpitas, CA, USA), anti-Mullerian hormone (AMH; Elabscience Biotechnology, MA, USA), follicle-stimulating hormone (FSH; Abnova, Taipei, Taiwan), and active caspase-3 (Mybiosource, San Diego, CA, USA) activity in serum were analyzed by ELISA kits, following the manufacturer's instructions. In brief, an equal volume of sample was added into the specific antibody-coated plates. Next, the specific horseradish peroxidase (HRP)-conjugates were added to each well and incubated at $37^{\circ} \mathrm{C}$. After the substrates had been added and incubated in the dark for substrate development, the antibody activity was analyzed by using a microplate reader (BioTek, Winooski, VT, USA). All samples were tested three times and the results are presented as the relative value.

\subsection{Genomic DNA Isolation}

To conduct genomic DNA (gDNA) isolation, the ovary tissues of rats were homogenized in LN2 and isolated by proteinase K (Qiagen) and lysis buffer, including radioimmunoprecipitation assay buffer (RIPA) buffer (Sigma-Aldrich), phosphatase inhibitor (Roche Diagnostics, Manheim, Germany), and protease inhibitor (A.G. Scientific, San Diego, CA, USA), for $4 \mathrm{~h}$ at $55{ }^{\circ} \mathrm{C}$. Next, phenol:chloroform:isoamyl alcohol (25:24:1; Sigma-Aldrich) was added to ligated samples and collected by centrifugation at 13,000 rpm for $30 \mathrm{~min}$. After this processing had been repeated twice, the digestion buffer containing iso-amylalchol (Sigma-Aldrich) and 0.3 M sodium acetate were added into the samples and incubated at $-20{ }^{\circ} \mathrm{C}$ overnight. After this, the pellet of gDNA was washed with $70 \%$ cold ethanol and eluted using Tris-EDTA buffer. The gDNA concentration was measured by nanodrop analysis. The gDNA was analyzed by human-specific primers. Primer sequences for human-specific Alu sequences (Accession number U14573) were as follows: (Forward) 5'-GGA GGC TGA GGC AGG AGA A-3' and (Reverse) 5'-ATC TCG GCT CAC TGC AAC CT-3'. The relative mRNA expression of the human Alu sequence gene was normalized by the cycle threshold values of rat GAPDH.

\subsection{RNA Isolation and Quantitative Real-Time Polymerase Chain Reaction Analysis}

Total RNA was isolated from snap-frozen ovaries using TRIzol reagent (Ambion, Boston, MA, USA), according to the manufacturer's protocols. The isolated RNA concentration was quantified by using a nanodrop spectrophotometer (Thermo Scientific). A total of $1 \mu \mathrm{g}$ of RNA was reverse transcribed into cDNA using the Superscript kit (Invitrogen, Carlsbad, CA, USA) containing 20 pmol of oligodT, $10 \mathrm{mM}$ of DNTP mix, RNase out, and Superscript III reverse transcriptase. The cDNA synthesis conditions were $65^{\circ} \mathrm{C}$ for $5 \mathrm{~min}, 50^{\circ} \mathrm{C}$ for $1 \mathrm{~h}$, and $72{ }^{\circ} \mathrm{C}$ for $15 \mathrm{~min}$. Amplification of the cDNA was accomplished in triplicate using a real-time polymerase chain reaction (PCR; Bio-Rad Laboratories Inc., Berkeley, CA, USA) instrument in the presence of commercially available SYBR Green PRC Master mix (Roche). The mRNA amplification conditions included incubation at $95^{\circ} \mathrm{C}$ for $5 \mathrm{~min}$, followed by 40 cycles at $95^{\circ} \mathrm{C}$ for $5 \mathrm{sec}$ and $60^{\circ} \mathrm{C}$ for $30 \mathrm{sec}$. All reactions were performed in triplicate. Alu sequences (Accession number U14573) were as follows: (Forward) 5'-GGA GGC TGA GGC AGG AGA A-3' and (Reverse) 5'-ATC TCG GCT CAC TGC AAC CT-3'. The relative mRNA expression of the human Alu sequence gene was normalized by the cycle threshold values of rat GAPDH.

\subsection{Protein Isolation and Western Blot Analysis}

All tissues were pooled to ensure no variation between groups. The ovary tissues were lysed using a lysis buffer containing protease inhibitor (Roche) and phosphatase inhibitor (A.G. Scientific., Sandiego, CA, USA) with RIPA buffer (Sigma-Aldrich) and then centrifuged at 13,000 $\mathrm{g}$ for $15 \mathrm{~min}$ at $4{ }^{\circ} \mathrm{C}$. The supernatants were collected for the next western blot. The concentration of proteins 
was determined with BSA kits (Thermo Fisher). Next, the total amount of proteins was separated by $6-15 \%$ sodium dodecyl sulfate-polyacrylamide gel electrophoresis (SDS-PAGE) and transferred onto polyvinylidene difluoride membranes (PVDF; Bio-Rad). After being blocked with 4\% (w/v) bovine serum albumin (BSA; Amresco, Solon, OH, USA) in Tris-buffered saline (Elbio, Deajun, Korea) containing $0.1 \%$ Tween-20 (Elbio) for $1 \mathrm{~h}$ at room temperature, the PVDF membranes were incubated with the primary antibody overnight at $4{ }^{\circ} \mathrm{C}$. Primary antibodies included the following: $P 4 H B$ (1:1000; Abcam, UK Cambridge), HO-1 (1:500; Novus Biologicals, CO, USA), HO-2 (1:500; Novus), $\operatorname{Prdx} 1$ (1:500; Abcam), Prdx2 (1:1000; Abcam), catalase (1:1000; Abcam), SOD1 (1:500; Cell Signaling Technology, MA, USA), Lhx8 (1:500; Santacruz, Texas, USA), Nobox (1:1000, Santacruz), Nanos3 (1:500; Abcam), and GAPDH (1:5000; Abfrontier, Seoul, Korea). After incubation in the primary antibodies, membranes were washed with tris buffered saline-tween 20 (TBS-T) for $5 \times 3$ min and incubated with the secondary antibody (Rabbit: 1:20,000; Mouse: 1:10,000; Goat: 1:5000) for $1 \mathrm{~h}$ at room temperature. After washing the samples with TBS-T buffer for $5 \times 3 \mathrm{~min}$ at room temperature, the immunoreactivity was visualized by enhanced chemiluminescence using enhanced chemiluminescence (ECL) kits (Bio-Rad) and Chemi-doc (Bio-Rad).

\subsection{Ovarian Follicle Counting Using Hematoxylin and Eosin (HEE) Staining}

All ovary tissues were fixed with $10 \%$ neutral buffered formalin solution containing $1 \times$ phosphate-buffered saline (PBS), 37\% formaldehyde (Merck, Darmstadt, Germany), and distilled water overnight. The ovary tissues were dehydrated, embedded in paraffin, and cut into sections $(4-5 \mu \mathrm{m})$. To analyze the formation of ovarian follicles in the ovaries, the tissue section slide was stained with hematoxylin (DAKO, Carpinteria, CA, USA) and eosin. Images were captured by using a Nikon microscope at $\times 1$ and $\times 40$ magnifications. The follicles were counted in two sections, which were the total follicles, including primordial, primary, secondary, and preovulatory follicles, and the antral follicles.

\subsection{PKH67-Labeled PD-MSCs Tracking}

To analyze the location of transplanted PD-MSCs in the ovary, PD-MSCs with labeled PKH67 were transplanted into the OVX rat model. After this, the ovarian frozen block was cut into sections (6-7 $\mu \mathrm{m})$. After ovary tissues had been washed with $1 \times$ phosphate-buffered saline (PBS) buffer, sectioned ovary tissues were incubated with propidium iodide (PI; SIGMA) solution for $30 \mathrm{~min}$ at $37^{\circ} \mathrm{C}$. Next, the sectioned ovary tissues were washed with $1 \times$ PBS and mounted using a mounting solution (DAKO). The fluorescence signal was observed by confocal instruments. The images were obtained from three independent trials.

\subsection{Immunofluorescence Staining}

To analyze the superoxidase in the mitochondria of ovarian follicles, the ovarian frozen block was cut into sections $(6-7 \mu \mathrm{m})$. After the ovary tissues had been washed with hanks' balanced salt solution (HBSS; GIBCO-BRL) buffer, they were incubated with $3 \mu \mathrm{M}$ Mito SOX (Invitrogen, Carlsbad, CA, USA) and $100 \mathrm{nM}$ Mito Tracker (Invitrogen) for $40 \mathrm{~min}$ at $37^{\circ} \mathrm{C}$. Next, the ovary tissues were washed with HBSS and incubated with $0.5 \mu \mathrm{g} 4^{\prime}$,6-diamidino-2-phenylindole (DAPI; SIGAM-Aldrich) for $1 \mathrm{~min}$ at room temperature. After the reaction, the ovary tissues were washed and mounted by using a mounting solution (DAKO). The detection of superoxide (red fluorescence) and mitochondria (green fluorescence) was conducted using confocal instruments. All data and images were obtained from three independent trials.

\subsection{Immunohistochemistry}

Ovary tissues were fixed by 10\% neutral buffered formalin (BBC Chemical, Mount Vernon, WA, USA), embedded in paraffin, and sectioned with $6 \mu \mathrm{m}$ of the ovaries. Sectioned ovary tissues were deparaffinized in a $60{ }^{\circ} \mathrm{C}$ dry oven by xylene and ethanol. Deparaffinized tissues underwent antigen 
retrieval by an EDTA (eLbio, Seongnam-Si, Korea) reaction. After removing the unbound primary antibody, the tissues in slides were incubated with DAKO Real EnVision HRP Rabbit/Mouse secondary antibody (Dako) at room temperature for $1 \mathrm{~h}$. The slides were incubated with DAB and counterstained with hematoxylin (Dako). After the reaction, slides were rinsed with tap water. Slides underwent dehydration by ethanol and xylene. Tissues were analyzed by the 3D HISTECH program (The Digital Pathology Company, Budapest, Hungary).

\subsection{Statistical Analysis}

For each set of data, independent experiments were repeated at least three times, with data representing the mean \pm standard deviation of at least three experiments. The statistical differences between experimental groups were determined by a t-test. The results were considered significant at $p<0.05$.

\section{Results}

\subsection{Transplanted PD-MSCs Engraft into the Damaged Ovary of an Ovariectomized Rat Model}

As Figure 1A shows, the PKH67-labeled PD-MSCs were located near the follicles of Tx groups at 1 week (Figure 1A). The human Alu-sequence gDNA expression was analyzed in the OVX group. The gDNA expression of the $h A l u$-sequence gene indicates the engraftment of human PD-MSCs. Interestingly, the gDNA expression of the $h A l u$-sequence was remarkably increased at 1 week after PD-MSCs in the Tx group compared to the NTx group. However, human Alu-sequence expression was not detected in the NTx group ( $p<0.05$; Figure 1B). The mRNA expression of the human Alu-sequence was significantly increased at 1 and 3 weeks after PD-MSCs in the Tx group compared to the NTx group. The mRNA expression of the human Alu-sequence demonstrates the metabolic activity of the engrafted human PD-MSCs $(p<0.05$; Figure 1C). These results indicated the homing activity of PD-MSCs to the ovary in the OVX model after PD-MSCs in the Tx group.

A
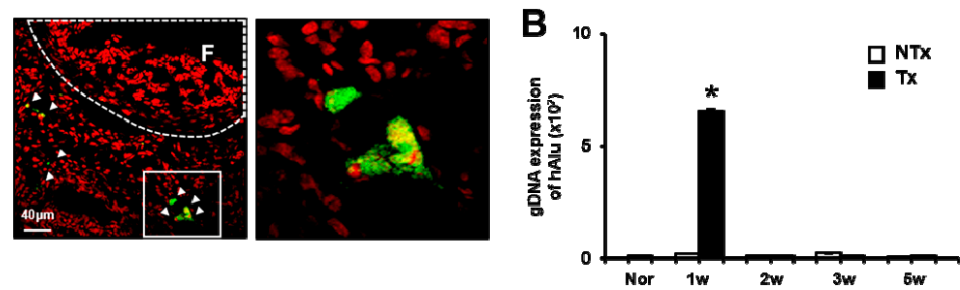

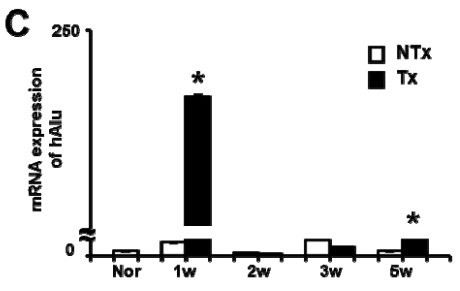

Figure 1. Placenta-derived mesenchymal stem cells (PD-MSCs) engrafted into the ovary of an ovariectomized rat model (OVX) rat model. The expression of PKH67-labeled PD-MSCs was localized in 1 week in the transplantation group (Tx) (A). The gDNA and mRNA expression levels of human Alu sequences in ovary tissues of the non-transplantation group (NTx) and Tx were analyzed by qRT-PCR $(B, C)$. The data are representative of three independent experiments and expressed as means \pm SD. * $p<0.05$ : NTx vs. Tx.

\subsection{The Analysis of Exosomes from Serum of the OVX Rat Model and the Expression of Antioxidant Factors}

To confirm the alteration of exosomes, we conducted an exosome analysis of the blood of the OVX rat model according to PD-MSCs Tx. As shown in Figure 2A,B, we identified the consequences of changing the exosomal components in the blood as stem cells are transplanted into the OVX rat model (Figure 2A,B). Interestingly, the exosome components in the blood exhibited changes in the expression of mitochondrial antioxidant factors, including energy metabolism and hormone and follicle development after PD-MSCs transplantation into the OVX rat model. Therefore, to analyze the antioxidant effect of mitochondria in the OVX rat model according to PD-MSCs Tx, we selected and compared the antioxidant factors among various factors and conducted the analysis at a protein level. Firstly, protein disulfide-isomerase $(P 4 H B)$, which is well-known as a molecular chaperone that 
generates ROS, was decreased in Tx compared to NTx by label-free quantitation using high-resolution LC-MS and western blot analyses (Figure 2C,D, Figure S1A). Secondly, catalase, which is an antioxidant enzyme that protects cells from oxidative stress, was significantly increased in Tx compared to NTx (Figure 2E,F, Figure S1B). Peredoxin1 and Peredoxin2 (Prdx1 and Prdx2), which are antioxidant enzymes that prevent oxidative damage, were remarkably increased in Tx compared to NTx (Figure 2G-J, Figure $\mathrm{S1C}$ ). These results indicated that transplanted PD-MSCs changed the microenvironment of the OVX rat model via the upregulated expression of various antioxidant enzymes.
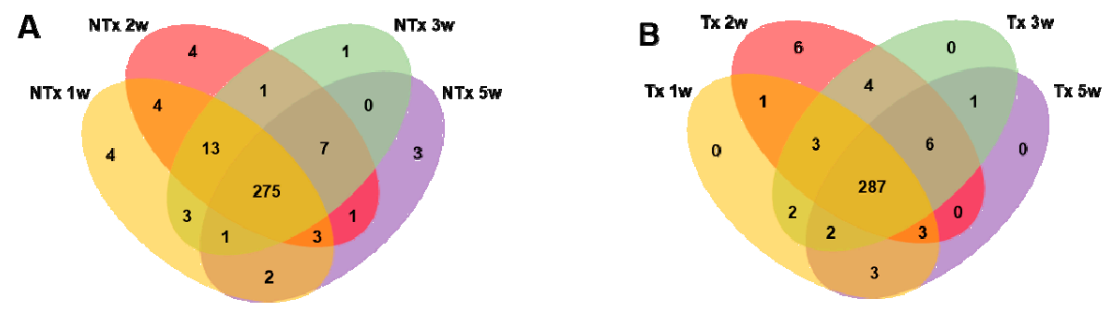

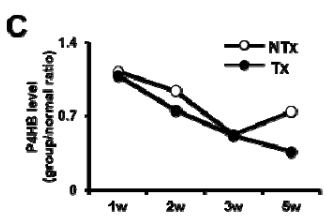

$\mathbf{G}$

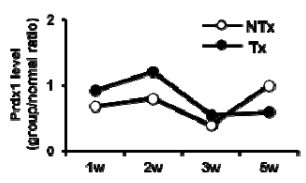

D

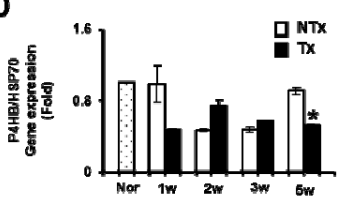

H

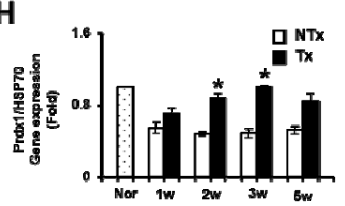

E

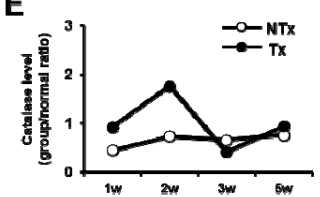

I

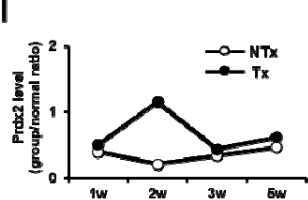

F

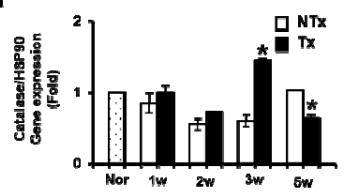

J

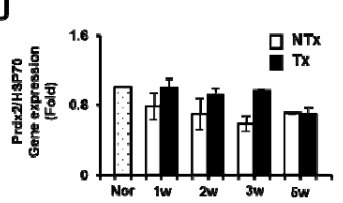

Figure 2. PD-MSCs trigger the upregulated expression of antioxidant enzymes in the exosome of the OVX rat model. The differences of factors in exosomes as a Venn diagram $(\mathbf{A}, \mathbf{B})$. The $P 4 H B$ expression related to the reactive oxygen species (ROS) level was analyzed in the exosome of serum (C,D). The catalase (E,F), $\operatorname{Prdx1}(\mathbf{G}, \mathbf{H})$, and $\operatorname{Prdx} 2(\mathbf{I}, \mathbf{J})$ expressions related to antioxidants were analyzed in the exosome of serum. The data are representative of three independent experiments and expressed as means \pm SD. ${ }^{*} p<0.05$ : NTx vs. Tx.

\subsection{PD-MSCs Transplantation Reduced Oxidative sTress and Enhanced the Antioxidant Effect in an Ovariectomized Rat Model}

As shown in Figure 3, the fluorescence intensities of MitoSOX in the ovary of the NTx group were higher compared to those in the Tx group. Those of the ovary of the Tx group exhibited the lowest accumulation of superoxide in the mitochondria and oocyte (Figure 3A). As a result of measuring the ratio between MitoSOX and MitoTracker, the ratio of MitoSOX/MitoTracker of antral follicles in the NTx group was shown to be remarkably increased compared to in the normal and Tx groups. In addition, the ratio of MitoSOX/ MitoTracker of the Tx group exhibited a lower expression in antral follicles compared to the NTx group ( $p<0.05$, Figure 3B).

Next, we analyzed heme oxygenase- 1 and -2 (HO-1 and HO-2) protein expression in the ovary of OVX groups according to PD-MSCs transplantation. The expressions of HO-1 and HO-2 genes were induced by oxidative stress. The gene expression of HO-1 in Tx groups was increased compared to that in NTx groups. The gene expression of HO-2 was significantly increased at 1 and 2 weeks after PD-MSCs transplantation compared to in the NTx group. However, HO-2 protein expression was significantly decreased at 3 and 5 weeks after PD-MSCs transplantation compared to that of the NTx group $(p<0.05$; Figure 3C). 

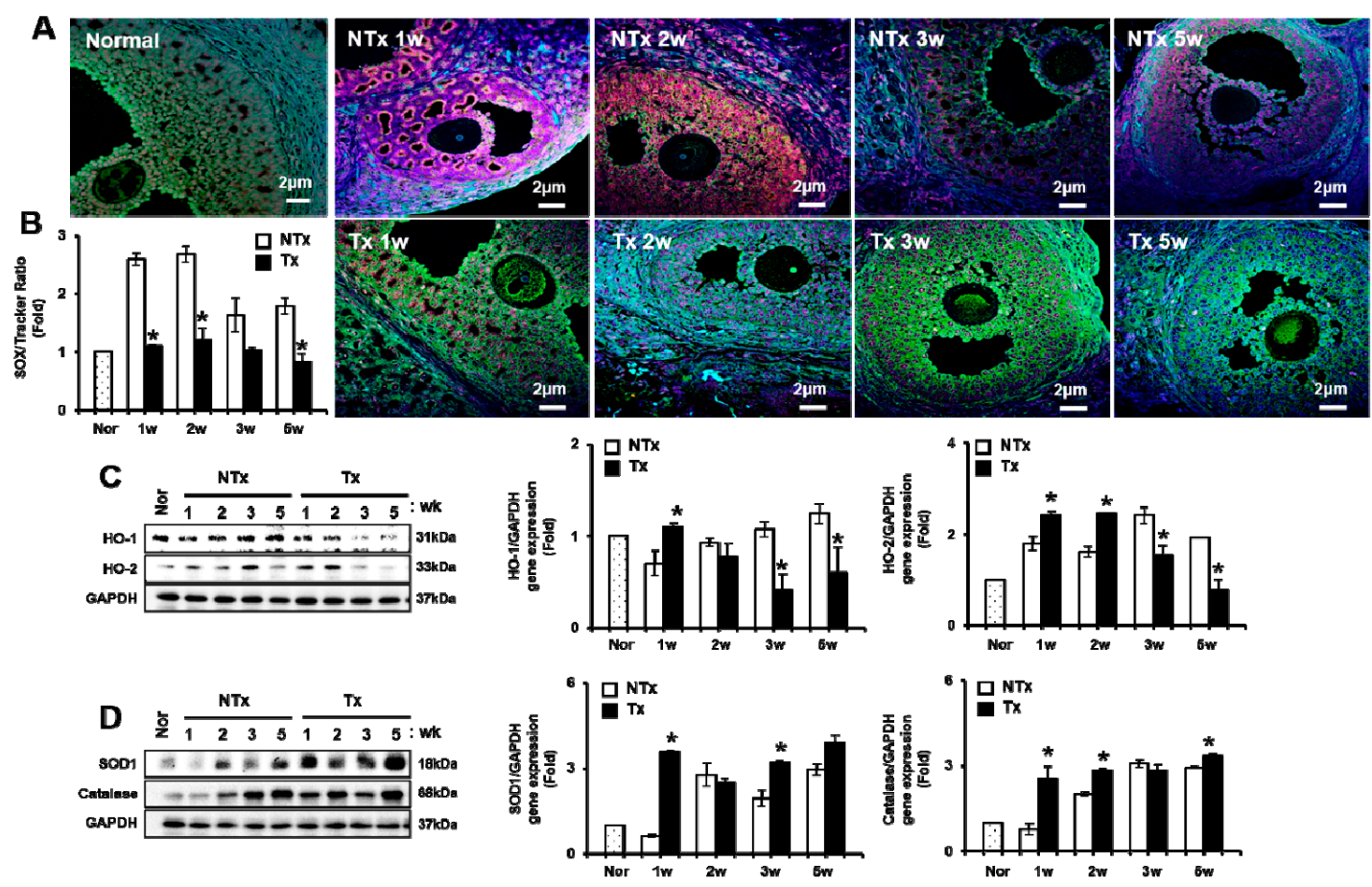

Figure 3. Effect of PD-MSCs transplantation on antioxidants in ovary tissues of the OVX rat model. The superoxide (Mito SOX) and mitochondria (Mito Tracker) in the ovary were stained by immunofluorescence (A) and measured by the Image J program (B). HO-1 and HO-2 gene expression related to oxidative induced factors $(\mathrm{C})$ and SOD1 and catalase gene expression related to antioxidant factors (D) were analyzed by western blot. The data are representative of three independent experiments and expressed as means \pm SD. ${ }^{*} p<0.05$ : NTx vs. Tx3.4. PD-MSCs Transplantation Inhibited Apoptosis in an Ovariectomized Rat Model

The protein expression of superoxide dismutase (SOD1), which is an enzyme that alternately catalyzes the dismutation of superoxide radicals, was analyzed in the OVX model. The gene expression of SOD1 was significantly increased in 1 week in the Tx group compared to the NTx group $(p<0.05$, Figure 3D). The gene expression of catalase, which is related to protecting the cell from oxidative damage by ROS, was investigated in the OVX model. The gene expression of catalase was significantly increased after PD-MSCs in Tx groups compared to that of NTx groups ( $p<0.05$, Figure 3D). These results suggested that PD-MSCs transplantation inhibited ROS production in mitochondria in ovary tissues through enhanced antioxidant factors in the OVX rat model.

Generally, it is important to maintain the balance between proliferation and cell death in organ development, as well as function. Therefore, we analyzed the proliferating cell nuclear antigen (PCNA) expression related to proliferation in follicles of the ovary of the rat, regardless of PD-MSCs transplantation. As shown in Figure 4A, PCNA positive signals in follicles were remarkably decreased in the NTx group compared to the control. However, PCNA positive signals in follicles of the Tx group were increased compared to those of the NTx group (Figure 4A). The results of the ELISA assay demonstrated that the activated caspase-3 activity was decreased after PD-MSCs in the Tx group compared to NTx groups. In particular, the activated caspase- 3 activity was significantly decreased at 1 and 3 weeks after PD-MSCs in Tx compared to the NTx groups $(p<0.05$, Figure 4B). The gene expression of Bcl2 in the Tx group showed the tendency to increase at 1 and 3 weeks compared to that of the NTx group (Figure 4C). The gene expression of cytochrome $C$ in the Tx group was significantly decreased at 3 and 5 weeks compared to that of the NTx group $(p<0.05$, Figure $4 \mathrm{D})$. These results suggest that PD-MSCs induce anti-apoptotic events in ovary tissues of the OVX model. 


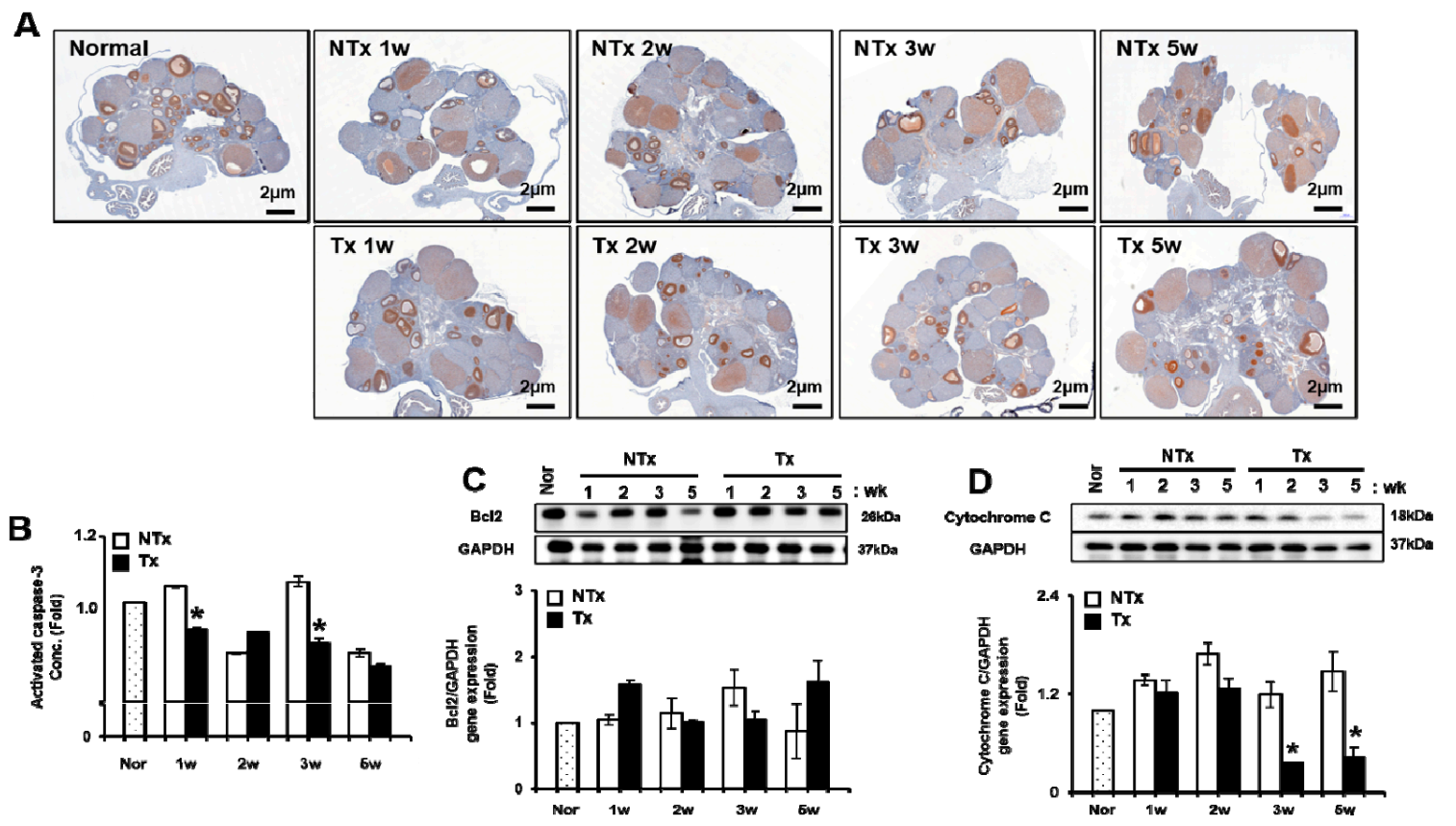

Figure 4. Effect of PD-MSCs transplantation on apoptosis in the ovary tissues of the OVX rat model. The PCNA expression in follicles of the ovary was stained by immunohistochemistry (A). The activated caspase-3 levels in the serum of NTx and Tx groups were analyzed by the ELISA assay (B). Bcl2 and cytochrome $C$ gene expression were analyzed by western blot $(\mathbf{C}, \mathbf{D})$. The data are representative of three independent experiments and expressed as means \pm SD. ${ }^{*} p<0.05$ : NTx vs. Tx.

\subsection{PD-MSCs Transplantation Enhanced the Ovarian Function through Follicular Development and Hormone} Expression in an Ovariectomized Rat Model

The ovarian function can be predicted by follicular development and hormonal changes. Therefore, we analyzed the hormone levels of anti-Mullerian hormone (AMH) and estrogen (E2), which are representative predictive markers for the ovarian function, in the blood of the OVX model, regardless of PD-MSCs transplantation, using ELISA assay kits. In particular, the AMH levels of serum, which have key roles in follicular growth and folliculogenesis, were decreased in the NTx group compared to normal groups after ovariectomy. Interestingly, the AMH levels of serum were significantly increased in the Tx group at 5 weeks after PD-MSCs transplantation compared to those of the NTx group $(p<0.05$, Figure 5A). Additionally, the estrogen (E2) levels of serum, which included the release of luteinizing hormone, were decreased in the NTx group compared to normal groups after ovariectomy. However, the E2 levels of serum were significantly increased in the Tx group compared to normal groups after PD-MSCs transplantation ( $p<0.05$, Figure 5B).

A

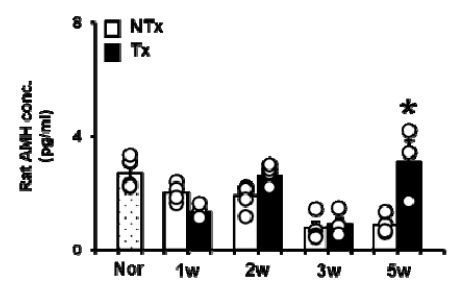

B

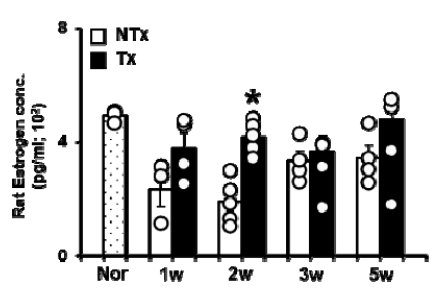

C

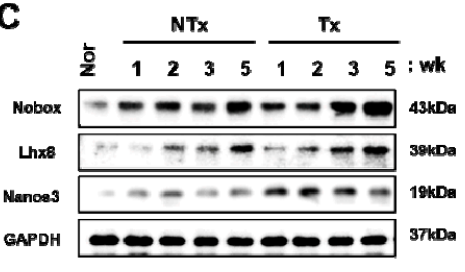

Figure 5. Effect of PD-MSCs transplantation on gene expression related to folliculogenesis in the ovary tissues of OVX rats. AMH and E2 hormone levels in the serum of NTx and Tx were analyzed by the ELISA assay (A,B). Nobox, Lhx8, and Nanos3 protein expression related to folliculogenesis in NTx and Tx were analyzed by Western blot $(\mathbf{C})$. The data are representative of three independent experiments and expressed as means \pm SD. ${ }^{*} p<0.05:$ NTx vs. Tx. 
To analyze the factors related to follicle development in the ovary of the OVX model, we analyzed the expression of the makers related to follicle development after PD-MSCs transplantation. The newborn ovary homeobox (Nobox) protein expression, which was expressed in the oocyte, was increased after PD-MSCs transplantation compared to NTx groups. The expression of LIM homeobox protein $8(\operatorname{Lh} x 8)$, which is an essential transcription factor of oogenesis processing, was increased in the Tx group compared to the NTx group. The expression of the Nanos homolog 3 (Nanos3) protein related to germ cell development was significantly increased at 1, 2, and 3 weeks after PD-MSCs transplantation compared to the NTx group ( $p<0.05$, Figure $5 \mathrm{C}$ and Figure S2). These results indicate that PD-MSCs transplantation can improve hormone levels (e.g., AMH and E2) and follicular growth in a rat model with ovariectomy.

\subsection{PD-MSC Transplantation Improved Follicular Development in an Ovariectomized Rat Model}

We confirmed the number of follicles in the OVX model after PD-MSCs transplantation. As Figure 6A shows, the number of follicles in the ovary was increased in all Tx groups (Figure 6A). To quantify the follicles according to each stage of maturity, primordial to antral follicles were measured by quoting Myers et al. [38]. The total follicles in NTx groups were significantly decreased compared to the normal group after ovariectomy. In particular, the number of primordial follicles compared to antral follicles was significantly decreased in the NTx group compared to those of the normal group; otherwise, the total follicles in the Tx group were remarkably increased $\left({ }^{*}{ }^{*} p<0.05\right.$, Figure $\left.6 \mathrm{~B}\right)$. The number of primordial follicles compared to antral follicles was extremely increased in the Tx group compared to those of the NTx group $\left({ }^{*},{ }^{* *} p<0.05\right.$, Figure $\left.6 \mathrm{~B}\right)$. These results indicate that PD-MSCs transplantation improves the total number of follicles decreased by ovariectomy and maturates the primordial follicles to antral follicles.

A

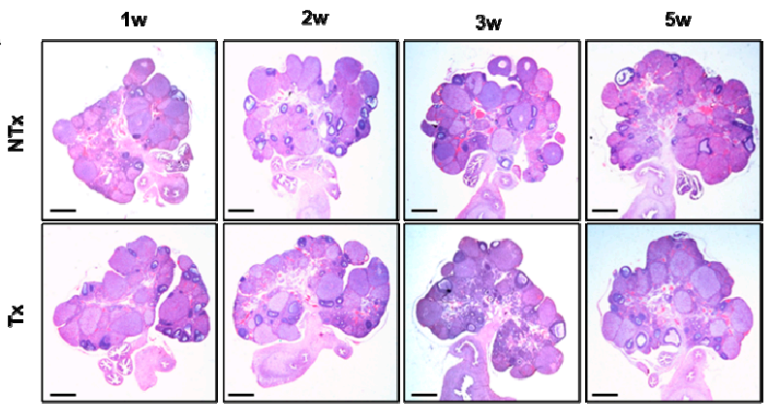

B

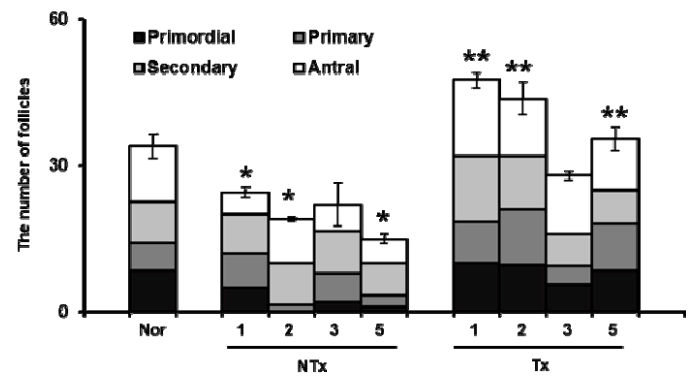

Figure 6. Effect of PD-MSCs transplantation on follicular development of the OVX rat model. Histopathological staining of ovary tissues was analyzed by hematoxylin and eosin staining in NTx and Tx (magnification $\times 10)(A)$. The numbers of total follicles and antral follicles were counted in NTx and $\operatorname{Tx}(\mathbf{B})$ The data are representative of three independent experiments and expressed as means \pm SD. $p<0.05:{ }^{*}$ Normal vs. NTx, ${ }^{* *}$ NTx vs. Tx.

\section{Discussion}

POF appears in women aged 40-50 years and is characterized by sex hormone deficiency, a loss of follicular activity, increasing atretic follicles, and infertility [39]. These risk factors are still not sufficiently known, although several studies have reported a correlation between risk factors and ovary dysfunction. Therefore, in this study, we focused on the efficacy of PD-MSCs in follicular development in an OVX rat model through antioxidant effects.

In recent years, the therapeutic effect of stem cells has been reported in degenerative diseases, including ovary dysfunction. Several previous studies have demonstrated that various stem cells improved the ovarian function, including folliculogenesis, sex hormone levels, and the ovarian niche [40-42]. BM-MSCs and amniotic epithelial cells (AECs) in an ovary dysfunction model inhibited apoptosis signaling in granulosa cells and restored the ovary function in chemotherapy-induced 
models $[43,44]$. Li et al. reported that human chorionic plate-derived MSCs (CP-MSCs) isolated from a normal-term placenta restored the ovarian function in a cyclophosphamide-induced POF mouse model [45]. Liu et al. and their colleagues demonstrated that BM-MSCs have an effect of homing and restored the function in an ovary dysfunctional model [46]. In our previous study, we demonstrated that an engrafted 3D spheroid type of PD-MSCs improved the ovarian function via upregulated gene expression related to folliculogenesis, as well as maturation follicles, in an OVX rat model [19]. These reports suggest that engrafted MSCs enhance the ovarian function through increasing the expressions of target genes related to folliculogenesis, as well as anti-apoptosis and the homing activity of MSCs. In this study, we also confirmed that PKH67-labeled PD-MSCs engrafted into ovary tissues of the OVX rat model after the intravenous transplantation of PD-MSCs, as well as hAlu expression in mRNA of ovary tissues (Figure 1). Therefore, this data demonstrates that transplanted PD-MSCs display homing activity in an ovary injured by ovariectomy through intravenous transplantation. This ability of MSCs is one of their major characteristics and correlates with their therapeutic efficacy in degenerative diseases. We confirmed that transplanted PD-MSCs trigger an upregulated gene expression related to folliculogenesis and hormone levels (e.g., AMH and E2) in the ovary of the OVX rat model (Figures 5 and 6). This data also indicates that the transplanted PD-MSCs improved ovarian function through antioxidants effects and anti-apoptosis.

The exosome can modulate the organ function of dysfunctional disease because it can produce passive movement in body fluids. Previous reports suggest that exosomes regulate the function of the ovary through cell to cell communication [47]. Hence, many researchers have reported that exosomes derived from MSCs have treatment potentials. Han et al. demonstrated that exosomes suppress apoptosis, including caspase-3 activation, via reduced mitochondrial membrane potential loss [48]. Sun et al. reported that exosomes derived from UC-MSCs suppress stress and apoptosis of granulosa cells by cisplatin [49]. Recently, it has been reported that exosomes secreted from ROS-exposed cells include antioxidant components for the defense of oxidative stress and also trigger tolerance to oxidative stress $[25,50,51]$. However, the study of microenvironmental change by MSCs is still not understood in ovarian dysfunctional models. Therefore, we confirmed whether the microenvironment in vivo is controlled by PD-MSCs transplantation. Firstly, we performed exosome profiling by LC-MS in the blood of OVX rat models after PD-MSCs transplantation. As results, the various antioxidants factors in exosomes from serum were found in serum after PD-MSCs transplantation (Figure 2). Among the several components in exosomes, selected antioxidant factors (e.g., catalase, and Prdx-1/-2) were increased by PD-MSCs transplantation. The expression of catalase was also increased in blood as well as ovary tissues after PD-MSCs transplantation. The selected factors are involved in the mitochondrial function and protect cell death through decreasing the ROS level in mitochondria of follicles in ovary tissues [52,53].

Oxidative stress-induced ROS result in cellular apoptosis in granulosa cells [54,55]. Several studies have reported a correlation between ROS levels and ovary dysfunction [56]. Shen et al. and their colleague reported that oxidative stress-induced apoptosis is associated with ovarian dysfunction by granulosa cell death. Furthermore, oxidative stress-induced apoptosis causes follicular atresia through Foxo1 overexpression [57]. Lee et al. demonstrated that the gene expression of HO-1 is increased by depleted glutathione in the ovary and induced oxidative stress by deficient estrogen levels in ovariectomized rat models [58]. In our study, the ROS levels were remarkably decreased in the Tx group compared to those of the NTx group through HO-1/-2 gene expression. Antioxidant markers (e.g., SOD1 and catalase) were significantly increased in the Tx group compared to the NTx group (Figure 3). Therefore, these previous reports are well-matched with our results and support our data. Finally, PD-MSCs regulate the microenvironment for protect oxidative stress in injured ovary tissues and restore the ovarian function in an OVX rat model through follicular development by antioxidants and an anti-apoptotic effect. 


\section{Conclusions}

The transplanted PD-MSCs migrated to the follicular periphery of the damaged ovarian tissue and improved the antioxidant efficacy by altering the HO-1/HO-2 expression in ovarian tissues and by increasing SOD1 and catalase gene expression. These results suggest that PD-MSCs transplanted into ovarian dysfunctional animal models reduce oxidative stress and apoptosis in injured ovarian tissue. Moreover, engrafted PD-MSCs trigger a restored ovary function, including increased gene expression related to follicle development, E2 and FSH hormone levels, and engagement in follicular maturation. These findings offer new insights for further understanding of stem cell therapy for reproductive systems and should provide new avenues to develop more efficient therapies in degenerative medicine.

Supplementary Materials: Supplementary materials can be found at http://www.mdpi.com/2076-3921/9/7/591/s1. Figure S1: PD-MSCs trigger an upregulated expression of antioxidant enzymes in the exosome of an OVX rat model, Figure S2: Effect of PD-MSCs transplantation on gene expression related to folliculogenesis in ovary tissues of OVX rats.

Author Contributions: Conceptualization, J.S. and G.J.K.; validation, investigation and formal analysis, J.S., H.P., J.H.C.; exosome analysis and data discussion, K.G.K.; image data analysis, J.-Y.L.; writing-review and editing, project administration, and funding acquisition, G.J.K. All authors have read and agreed to the published version of the manuscript.

Funding: This work was supported by the Basic Science Research Program through the National Research Foundation of Korea (NRF) funded by the Ministry of Science, ICT \& Future Planning (2017M3A9B4061665).

Conflicts of Interest: The authors declare no conflict of interest.

\section{Abbreviations}

PD-MSCs: Placenta-derived mesenchymal stem cells; OVX: ovariectomized rat model; ROS: reactive oxygen species; HO-1: heme oxygenase-1; HO-2: heme oxygenase-2; SOD1: superoxide dismutase; CAT: catalase; Lhx 8: Lim homebox 8; FSH: follicle-stimulating hormone; AMH: anti-Mullerian hormone.

\section{References}

1. Sukur, Y.E.; Kivancli, I.B.; Ozmen, B. Ovarian aging and premature ovarian failure. J. Turk. Ger. Gynecol. Assoc. 2014, 15, 190-196. [CrossRef] [PubMed]

2. Vabre, P.; Gatimel, N.; Moreau, J.; Gayrard, V.; Picard-Hagen, N.; Parinaud, J.; Leandri, R.D. Environmental pollutants, a possible etiology for premature ovarian insufficiency: A narrative review of animal and human data. Environ. Health 2017, 16, 37. [CrossRef] [PubMed]

3. De Vos, M.; Devroey, P.; Fauser, B.C. Primary ovarian insufficiency. Lancet 2010, 376, 911-921. [CrossRef]

4. Collins, G.; Patel, B.; Thakore, S.; Liu, J. Primary Ovarian Insufficiency: Current Concepts. South Med. J. 2017, 110, 147-153. [CrossRef] [PubMed]

5. Dragojevic-Dikic, S.; Marisavljevic, D.; Mitrovic, A.; Dikic, S.; Jovanovic, T.; Jankovic-Raznatovic, S. An immunological insight into premature ovarian failure (POF). Autoimmun. Rev. 2010, 9, 771-774. [CrossRef] [PubMed]

6. Sullivan, S.D.; Sarrel, P.M.; Nelson, L.M. Hormone replacement therapy in young women with primary ovarian insufficiency and early menopause. Fertil. Steril. 2016, 106, 1588-1599. [CrossRef]

7. Tavani, A.; La Vecchia, C. The adverse effects of hormone replacement therapy. Drugs Aging 1999, 14, 347-357. [CrossRef]

8. Silber, S. Ovarian tissue cryopreservation and transplantation: Scientific implications. J. Assist. Reprod. Genet. 2016, 33, 1595-1603. [CrossRef]

9. Kim, M.J.; Shin, K.S.; Jeon, J.H.; Lee, D.R.; Shim, S.H.; Kim, J.K.; Cha, D.H.; Yoon, T.K.; Kim, G.J. Human chorionic-plate-derived mesenchymal stem cells and Wharton's jelly-derived mesenchymal stem cells: A comparative analysis of their potential as placenta-derived stem cells. Cell Tissue Res. 2011, 346, 53-64. [CrossRef]

10. Seok, J.; Jung, H.S.; Park, S.; Lee, J.O.; Kim, C.J.; Kim, G.J. Alteration of fatty acid oxidation by increased CPT1A on replicative senescence of placenta-derived mesenchymal stem cells. Stem. Cell Res. Ther. 2020, 11, 1. [CrossRef] 
11. Lee, J.M.; Jung, J.; Lee, H.J.; Jeong, S.J.; Cho, K.J.; Hwang, S.G.; Kim, G.J. Comparison of immunomodulatory effects of placenta mesenchymal stem cells with bone marrow and adipose mesenchymal stem cells. Int. Immunopharmacol. 2012, 13, 219-224. [CrossRef] [PubMed]

12. Thummasorn, S.; Kumfu, S.; Chattipakorn, S.; Chattipakorn, N. Granulocyte-colony stimulating factor attenuates mitochondrial dysfunction induced by oxidative stress in cardiac mitochondria. Mitochondrion 2011, 11, 457-466. [CrossRef]

13. Lee, M.J.; Jung, J.; Na, K.H.; Moon, J.S.; Lee, H.J.; Kim, J.H.; Kim, G.I.; Kwon, S.W.; Hwang, S.G.; Kim, G.J. Anti-fibrotic effect of chorionic plate-derived mesenchymal stem cells isolated from human placenta in a rat model of $\mathrm{CCl}(4)$-injured liver: Potential application to the treatment of hepatic diseases. J. Cell Biochem. 2010, 111, 1453-1463. [CrossRef] [PubMed]

14. Jung, J.; Choi, J.H.; Lee, Y.; Park, J.W.; Oh, I.H.; Hwang, S.G.; Kim, K.S.; Kim, G.J. Human placenta-derived mesenchymal stem cells promote hepatic regeneration in CCl4-injured rat liver model via increased autophagic mechanism. Stem. Cells 2013, 31, 1584-1596. [CrossRef]

15. Jung, J.; Moon, J.W.; Choi, J.H.; Lee, Y.W.; Park, S.H.; Kim, G.J. Epigenetic Alterations of IL-6/STAT3 Signaling by Placental Stem Cells Promote Hepatic Regeneration in a Rat Model with CCl4-induced Liver Injury. Int. J. Stem. Cells 2015, 8, 79-89. [CrossRef]

16. Chung, S.; Rho, S.; Kim, G.; Kim, S.R.; Baek, K.H.; Kang, M.; Lew, H. Human umbilical cord blood mononuclear cells and chorionic plate-derived mesenchymal stem cells promote axon survival in a rat model of optic nerve crush injury. Int. J. Mol. Med. 2016, 37, 1170-1180. [CrossRef]

17. Kim, J.Y.; Jun, J.H.; Park, S.Y.; Yang, S.W.; Bae, S.H.; Kim, G.J. Dynamic Regulation of miRNA Expression by Functionally Enhanced Placental Mesenchymal Stem Cells PromotesHepatic Regeneration in a Rat Model with Bile Duct Ligation. Int. J. Mol. Sci. 2019, 20, 5299. [CrossRef] [PubMed]

18. Lee, Y.B.; Choi, J.H.; Kim, E.N.; Seok, J.; Lee, H.J.; Yoon, J.H.; Kim, G.J. Human Chorionic Plate-Derived Mesenchymal Stem Cells Restore Hepatic Lipid Metabolism in a Rat Model of Bile Duct Ligation. Stem. Cells Int. 2017, 2017, 5180579. [CrossRef] [PubMed]

19. Kim, T.H.; Choi, J.H.; Jun, Y.; Lim, S.M.; Park, S.; Paek, J.Y.; Lee, S.H.; Hwang, J.Y.; Kim, G.J. 3D-cultured human placenta-derived mesenchymal stem cell spheroids enhance ovary function by inducing folliculogenesis. Sci. Rep. 2018, 8, 15313. [CrossRef]

20. Kalluri, R.; LeBleu, V.S. The biology, function, and biomedical applications of exosomes. Science 2020, 367, 6478. [CrossRef]

21. Taylor, D.D.; Gercel-Taylor, C. The origin, function, and diagnostic potential of RNA within extracellular vesicles present in human biological fluids. Front. Genet. 2013, 4, 142. [CrossRef] [PubMed]

22. Maia, J.; Caja, S.; Strano Moraes, M.C.; Couto, N.; Costa-Silva, B. Exosome-Based Cell-Cell Communication in the Tumor Microenvironment. Front. Cell Dev. Biol. 2018, 6, 18. [CrossRef] [PubMed]

23. Javeed, N.; Mukhopadhyay, D. Exosomes and their role in the micro-/macro-environment: A comprehensive review. J. Biomed. Res. 2017, 31, 386-394.

24. Feng, W.; Dean, D.C.; Hornicek, F.J.; Shi, H.; Duan, Z. Exosomes promote pre-metastatic niche formation in ovarian cancer. Mol. Cancer 2019, 18, 124. [CrossRef]

25. Eldh, M.; Ekstrom, K.; Valadi, H.; Sjostrand, M.; Olsson, B.; Jernas, M.; Lotvall, J. Exosomes communicate protective messages during oxidative stress; possible role of exosomal shuttle RNA. PLoS ONE 2010, 5, e15353. [CrossRef] [PubMed]

26. Jezek, P.; Holendova, B.; Plecita-Hlavata, L. Redox Signaling from Mitochondria: Signal Propagation and Its Targets. Biomolecules 2020, 10, 93. [CrossRef] [PubMed]

27. Birben, E.; Sahiner, U.M.; Sackesen, C.; Erzurum, S.; Kalayci, O. Oxidative stress and antioxidant defense. World Allergy Organ. J. 2012, 5, 9-19. [CrossRef]

28. Halliwell, B. Tell me about free radicals, doctor: A review. J. R. Soc. Med. 1989, 82, 747-752. [CrossRef]

29. Kudryavtseva, A.V.; Krasnov, G.S.; Dmitriev, A.A.; Alekseev, B.Y.; Kardymon, O.L.; Sadritdinova, A.F.; Fedorova, M.S.; Pokrovsky, A.V.; Melnikova, N.V.; Kaprin, A.D.; et al. Mitochondrial dysfunction and oxidative stress in aging and cancer. Oncotarget 2016, 7, 44879-44905. [CrossRef]

30. Hori, Y.S.; Kuno, A.; Hosoda, R.; Horio, Y. Regulation of FOXOs and p53 by SIRT1 modulators under oxidative stress. PLoS ONE 2013, 8, e73875. [CrossRef]

31. Shkolnik, K.; Tadmor, A.; Ben-Dor, S.; Nevo, N.; Galiani, D.; Dekel, N. Reactive oxygen species are indispensable in ovulation. Proc. Natl. Acad. Sci. USA 2011, 108, 1462-1467. [CrossRef] [PubMed] 
32. Agarwal, A.; Aponte-Mellado, A.; Premkumar, B.J.; Shaman, A.; Gupta, S. The effects of oxidative stress on female reproduction: A review. Reprod. Biol. Endocrinol. 2012, 10, 49. [CrossRef]

33. Wojsiat, J.; Korczynski, J.; Borowiecka, M.; Zbikowska, H.M. The role of oxidative stress in female infertility and in vitro fertilization. Postep. Hig. Med. Dosw. (Online) 2017, 71, 359-366. [CrossRef] [PubMed]

34. Lai, Q.; Xiang, W.; Li, Q.; Zhang, H.; Li, Y.; Zhu, G.; Xiong, C.; Jin, L. Oxidative stress in granulosa cells contributes to poor oocyte quality and IVF-ET outcomes in women with polycystic ovary syndrome. Front. Med. 2018, 12, 518-524. [CrossRef] [PubMed]

35. Prasad, S.; Tiwari, M.; Pandey, A.N.; Shrivastav, T.G.; Chaube, S.K. Impact of stress on oocyte quality and reproductive outcome. J. Biomed. Sci. 2016, 23, 36. [CrossRef] [PubMed]

36. Zougman, A.; Selby, P.J.; Banks, R.E. Suspension trapping (STrap) sample preparation method for bottom-up proteomics analysis. Proteomics 2014, 14, 1006. [CrossRef]

37. Nolte, H.; MacVicar, T.D.; Tellkamp, F.; Kruger, M. Instant Clue: A Software Suite for Interactive Data Visualization and Analysis. Sci. Rep. 2018, 8, 12648. [CrossRef]

38. Myers, M.; Britt, K.L.; Wreford, N.G.; Ebling, F.J.; Kerr, J.B. Methods for quantifying follicular numbers within the mouse ovary. Reproduction 2004, 127, 569-580. [CrossRef]

39. Jankowska, K. Premature ovarian failure. Prz. Menopauzalny 2017, 16, 51-56. [CrossRef]

40. Song, D.; Zhong, Y.; Qian, C.; Zou, Q.; Ou, J.; Shi, Y.; Gao, L.; Wang, G.; Liu, Z.; Li, H.; et al. Human Umbilical Cord Mesenchymal Stem Cells Therapy in Cyclophosphamide-Induced Premature Ovarian Failure Rat Model. Biomed. Res. Int. 2016, 2016, 2517514. [CrossRef]

41. Elfayomy, A.K.; Almasry, S.M.; El-Tarhouny, S.A.; Eldomiaty, M.A. Human umbilical cord blood-mesenchymal stem cells transplantation renovates the ovarian surface epithelium in a rat model of premature ovarian failure: Possible direct and indirect effects. Tissue Cell 2016, 48, 370-382. [CrossRef] [PubMed]

42. Fu, X.; He, Y.; Xie, C.; Liu, W. Bone marrow mesenchymal stem cell transplantation improves ovarian function and structure in rats with chemotherapy-induced ovarian damage. Cytotherapy 2008, 10, 353-363. [CrossRef] [PubMed]

43. Guo, J.Q.; Gao, X.; Lin, Z.J.; Wu, W.Z.; Huang, L.H.; Dong, H.Y.; Chen, J.; Lu, J.; Fu, Y.F.; Wang, J.; et al. BMSCs reduce rat granulosa cell apoptosis induced by cisplatin and perimenopause. BMC Cell Biol. 2013, 14, 18. [CrossRef] [PubMed]

44. Zhang, Q.; Xu, M.; Yao, X.; Li, T.; Wang, Q.; Lai, D. Human amniotic epithelial cells inhibit granulosa cell apoptosis induced by chemotherapy and restore the fertility. Stem. Cell Res. Ther. 2015, 6, 152. [CrossRef]

45. Li, J.; Yu, Q.; Huang, H.; Deng, W.; Cao, X.; Adu-Frimpong, M.; Yu, J.; Xu, X. Human chorionic plate-derived mesenchymal stem cells transplantation restores ovarian function in a chemotherapy-induced mouse model of premature ovarian failure. Stem. Cell Res. Ther. 2018, 9, 81. [CrossRef]

46. Liu, J.; Zhang, H.; Zhang, Y.; Li, N.; Wen, Y.; Cao, F.; Ai, H.; Xue, X. Homing and restorative effects of bone marrow-derived mesenchymal stem cells on cisplatin injured ovaries in rats. Mol. Cells 2014, 37, 865-872. [CrossRef]

47. Popiolek, K.; Grzesiak, M. Exosomes as a new approach into cell-to-cell communication within the mammalian ovary. Postepy Biochem. 2019, 65, 263-270.

48. Han, S.; Rhee, W.J. Inhibition of apoptosis using exosomes in Chinese hamster ovary cell culture. Biotechnol. Bioeng. 2018, 115, 1331-1339. [CrossRef]

49. Sun, L.; Li, D.; Song, K.; Wei, J.; Yao, S.; Li, Z.; Su, X.; Ju, X.; Chao, L.; Deng, X.; et al. Exosomes derived from human umbilical cord mesenchymal stem cells protect against cisplatin-induced ovarian granulosa cell stress and apoptosis in vitro. Sci. Rep. 2017, 7, 2552. [CrossRef]

50. Saeed-Zidane, M.; Linden, L.; Salilew-Wondim, D.; Held, E.; Neuhoff, C.; Tholen, E.; Hoelker, M.; Schellander, K.; Tesfaye, D. Cellular and exosome mediated molecular defense mechanism in bovine granulosa cells exposed to oxidative stress. PLoS ONE 2017, 12, e0187569. [CrossRef]

51. Kala, M.; Shaikh, M.V.; Nivsarkar, M. Equilibrium between anti-oxidants and reactive oxygen species: A requisite for oocyte development and maturation. Reprod. Med. Biol. 2017, 16, 28-35. [CrossRef] [PubMed]

52. Mihalas, B.P.; Redgrove, K.A.; McLaughlin, E.A.; Nixon, B. Molecular Mechanisms Responsible for Increased Vulnerability of the Ageing Oocyte to Oxidative Damage. Oxid. Med. Cell Longev. 2017, 2017, 4015874. [CrossRef] [PubMed]

53. Lim, J.; Luderer, U. Oxidative damage increases and antioxidant gene expression decreases with aging in the mouse ovary. Biol. Reprod. 2011, 84, 775-782. [CrossRef] [PubMed] 
54. Chen, P.; Hu, Y.F.; Wang, L.; Xiao, W.F.; Bao, X.Y.; Pan, C.; Yi, H.S.; Chen, X.Y.; Pan, M.H.; Lu, C. Mitochondrial Apoptotic Pathway Is Activated by $\mathrm{H}_{2} \mathrm{O}_{2}$-Mediated Oxidative Stress in BmN-SWU1 Cells from Bombyx mori Ovary. PLoS ONE 2015, 10, e0134694. [CrossRef]

55. Yang, H.; Xie, Y.; Yang, D.; Ren, D. Oxidative stress-induced apoptosis in granulosa cells involves JNK, p53 and Puma. Oncotarget 2017, 8, 25310-25322. [CrossRef]

56. Jiang, H.L.; Cao, L.Q.; Chen, H.Y. Protective effects ROS up-regulation on premature ovarian failure by suppressing ROS-TERT signal pathway. Eur. Rev. Med. Pharmacol. Sci. 2018, 22, 6198-6204.

57. Shen, M.; Lin, F.; Zhang, J.; Tang, Y.; Chen, W.K.; Liu, H. Involvement of the up-regulated FoxO1 expression in follicular granulosa cell apoptosis induced by oxidative stress. J. Biol. Chem. 2012, 287, 25727-25740. [CrossRef]

58. Lee, Y.M.; Cheng, P.Y.; Hong, S.F.; Chen, S.Y.; Lam, K.K.; Sheu, J.R.; Yen, M.H. Oxidative stress induces vascular heme oxygenase-1 expression in ovariectomized rats. Free Radic Biol. Med. 2005, 39, 108-117. [CrossRef]

(C) 2020 by the authors. Licensee MDPI, Basel, Switzerland. This article is an open access article distributed under the terms and conditions of the Creative Commons Attribution (CC BY) license (http://creativecommons.org/licenses/by/4.0/). 